\title{
Concepto Actual del Fórceps
}

\author{
Dr. Rafael Ramirez Merchán \\ Del Instituto Materno-Infantil - Bogotá
}

Uno de los grandes acontecimientos de la medicina fue indudablemente el invento del Fórceps para extraer vivo al producto de la concepción, por las vías naturales con lo cual quedaban abolidas las intervenciones mutiladoras sobre feto vivo. Su importancia se agiganta si se recuerda que se ha utilizado desde mediados del siglo XVII y que los grandes progresos que significaron la asepsia, la antisepsia, la regulación de técnicas quirúrgicas, entre ellas la sutura de la matriz en la operación cesárea, son adquisiciones que tocan muy de cerca con el propio siglo XX. Es decir, que con haber significado mucho este instrumento, la suerte de la parturienta eutócica o distócica era sombrío, por todos los aspectos, hasta finales del siglo XIX.

La supervivencia fetal era cosa secundaria, o de antemano se descartaba ante la presencia de la distocia. Otro es el panorama de la época: el perfeccionamiento de técnicas quirúrgicas, los inverosímiles adelantos de la anestesiología, la asepsia y la antisepsia perfeccionadas, la infección dominada con los antibióticos, la medicina preventiva abarcando todas las posibilidades de su definición; el conocimiento pormenorizado de los fenómenos biológicos, el escudriñamiento de las lesiones anatomopatológicas de la enfermedad, los adelantos farmacológicos y la visión del conjunto en los registros estadísticos, fiscalizan todas y cada una de nuestras determinaciones profesionales y juzgan inexorablemente la actuación de quien se denomina especialista en determinada rama de las ciencias médicas.

En lo concerniente al ejercicio de la Clínica Obstétrica y específicamente al empleo del fórceps para solucionar determinadas distocias, el gran adelanto ha consistido en proceder siempre sin detrimento de la madre ni del hijo. Maravillémonos pues, de que todavía se utilice este instrumento que, indebidamente aplica- 
do puede ser una forma disimulada de fetotomía "in vivo" o una solución en falso que puede llevar a una supervivencia fetal pródiga en complicaciones neurológicas. Esto en cuanto al producto se refiere, porque la madre tampoco queda exenta de muy serias complicaciones.

Ninguna intervención obstétrica tiene hoy en día indicación si de antemano se sabe que ha de causar algún daño previsible a la madre o al hijo y quien ejerce en esta rama de la medicina ha de saber que su conducta tiene proyecciones en el futuro como acaba de mencionarse. La Obstetricia, la Ginecología y la Pediatría tienen correlaciones ineludibles y el mutuo entendimiento y la estrecha colaboración de los especialistas, son indispensables para su correcto ejercicio y para seguridad de los pacientes. Los conocimientos ginecológicos del obstetra deben actualizarse para no menospreciar la responsabilidad que le incumbe por acción u omisión en el oportuno diagnóstico de las entidades ginecológicas y en la previsión de las complicaciones a que pueda dar origen una conducta inconsulta. Por su parte la pediatría es la encargada de dar la argumentación para abolir determinadas maniobras o conductas obstétricas que no se justifican, por el alto grado de peligrosidad que en sí tienen, cuando hay intervenciones que ventajosamente las suplen.

Estas consideraciones dan la explicación de por qué hoy no sería correcto ni justificable que las aplicaciones del fórceps dieran algún porcentaje de mortalidad y que si alguna morbosidad sigue a la intervención, no podría ser otra que la inevitable en el parto espontáneo; y en cuanto al traumatismo, debe ser mínimo e intrascendente si con rigor inflexible se llenan los requisitos que la experiencia ha fijado como norma. Así idealizada esta intervención, requiere necesariamente conocimientos y experiencia que concurren en el momento de decidirla, y culminan en la serie de movimientos coordinados que suplen la falla fisiológica e imitan el mecanismo normal del parto. La pericia del operador se pone asaz de manifiesto cuando sabe desistir oportunamente si encuentra dificultades; porque si esto sucede a quien conoce la técnica, el fórceps no está indicado. Pudieran parecer exagerados o pedantes estos conceptos; mas si recordamos que el estudio de la patología del recién nacido da un apreciable porcentaje de morbosidad a la prolongación del tiempo de desprendimiento, (1) se comprende fácilmente no solo la necesidad de la intervención 
oportuna sino que el fórceps cumpla una acción profiláctica de la hemorragia meníngea y de serias cerebropatías consecutivas a la anoxia que ocurren con mayor frecuencia en el nacimiento de fetos prematuros. (2) La indicación profiláctica ha sido, en mi concepto, la que salvó al instrumento de sufrir el definitivo rechazo que sufrieron intervenciones tales como la dilatación manual del cuello, la gran extracción de pelvis y las maniobras de conversión de una presentación a otra.

Tiene pues el fórceps dos indicaciones fundamentales: una profiláctica y otra terapéutica. La primera cubre más del $70 \%$ del total de aplicaciones, porque es de rigor en las primerizas, no se descarta en las multíparas y es imperativo en el parto de la paciente cardíaca, de la tuberculosa, de la toxémica y en la paciente con antecedente de cesárea en quien se ha permitido el parto por las vías naturales. Para que una aplicación pueda llamarse profiláctica, estrictamente hablando, la presentación debe haber descendido y rotado a occipito-púbica y al practicar la episiotomía previa, la cabeza debe ser visible. (3) Si la presentación ha descendido pero no ha rotado a $\mathrm{O}$. P. la aplicación no sería profiláctica sino una aplicación baja que ya tiene riesgos y el más frecuente es el desgarro vaginal en tratándose de primerizas.

La indicación terapéutica del fórceps surge cuando cumplido completamente el primer período del parto, (período de dilatación), encajada y descendida la presentación, se detiene su progreso, o cuando es imperativo terminar aceleradamente el parto por causa materna o fetal. La detención del progreso ocurre en el más alto porcentaje de los casos, en las presentaciones posteriores (ODP - OIP) en las cuales no se hace la rotación espontánea o apenas se hace a OT por mala flexión. Es lo que los textos americanos denominan las occipito posteriores persistentes y la detención transversa profunda.

La intervención puede practicarse de manera electiva o también de urgencia por la súbita alteración de los ruidos cardíacos fetales o cualquier manifestación patológica de la madre que aconseja la terminación del parto. El sufrimiento fetal exige inmediatas medidas tendientes a propiciar una mejor oxigenación placentaria mientras se interviene de urgencia. La pericia del obstetra consiste en tomar una determinación adelantándosele al sufrimiento fetal y no esperar el sufrimiento para decidir una conducta. Segundos de anoxia pueden dejar lesiones cerebrales irrepa- 
rables. En tal sentido, como lo anota Lull (4) el fórceps que hemos denominado terapéutico tendría también una función profiláctica; pero no debe creerse que con él se puedan resolver siempre las distocias que comentamos; en algunos casos será más segura la operación cesárea. Y no debe establecerse emulación entre una y otra intervención porque una indicación justa de cesárea excluye al fórceps y la presencia de las condiciones favorables al fórceps, objetivamente consideradas, excluye la cesárea en primera instancia.

En efecto el fórceps alto está universalmente abandonado. Si al práctico le entra la tentación de aplicarlo, debe saber que está haciendo algo indebido; que va a exponer a graves riesgos tanto a la madre como al feto, que el fracaso ha sido previsto y que es otra la intervención que le dará el máximum de seguridades con el mínimum de peligros. Débese pues respetar la norma, no ya del encajamiento sino del encajamiento con descenso de la presentación.

Clínicamente la conveniencia del fórceps se aprecia por medio de los siguientes datos: la parte más baja del cráneo debe haber pasado en uno o 2 centímetros la línea imaginaria que une las dos espinas ciáticas. Esto se aprecia objetivamente porque la concavidad del sacro está ocupada totalmente por la presentación, solamente queda una cuarta parte del pubis libre y la cabeza se ha moldeado en forma de poder hacer un diagnóstico exacto de la variedad de posición. Si hay bosa serosanguínea debe descartarse su volumen en la apreciación del caso. En el lenguaje corriente se utiliza la denominación de De Lee (5) en la siguiente forma : cuando la parte más baja de la presentación está a la altura de la línea que une las dos espinas ciáticas se llama la estación 0 o sea, presentación en vía de encajamiento y los grados de encajamiento se expresan en más uno, más dos, más tres, más cuatro, más cinco, según los centímetros que haya descendido. Inversamente la altura de la presentación antes del encajamiento se expresa en menos uno, menos dos, menos tres, menos cuatro, menos cinco.

Mencionamos atrás que el cuello de la matriz es "tabú" en la obstetricia actual; su dilatación manual, su dilatación forzada, fué un recurso desesperado de épocas ya superadas. De consiguiente el cuello debe ser dilatado espontáneamente y, mejor aún, debe haberse hecho abdominal para que permita la aplica- 
ción del fórceps. Si este requisito se menosprecia, son seguras y graves las lesiones que se producirán tanto a la madre como al feto. El traumatismo causará lesiones irreparables al feto y para la madre tiene el peligro del desgarro cervical con posibilidades de propagación, hemoragia y shock. El diagnóstico exacto de la posición y su variedad, previa ruptura de las membranas, determinará el eje de aplicación según las normas clásicas para lograr una toma ideal, la menos traumatizante.

Técnica operatoria. - La regla más sencilla y segura para las aplicaciones de fórceps es la que aconsejan los autores franceses de hacer el proyecto de la misma teniendo en cuenta dos ejes: el que ocupa la sutura sagital y el que va a ocupar el fórceps; estos dos ejes van a cruzarse; es decir, si la presentación ocupa el diámetro oblicuo derecho el fórceps quedará aplicado en el diámetro oblicuo izquierdo y viceversa, en las aplicaciones sobre cabeza encajada, descendida y rotada a O.P. u O.S. que están ocupando el diámetro anteroposterior, el fórceps queda en el diámetro transverso. Es la toma y la aplicación más fácil, más sencilla, más segura y más correcta. (6)

Manejo de las variedades posteriores y transversas. - Quiero insistir sobre la indicación del fórceps y su manual operatorio en las variedades posteriores y transversas porque son éstas las que dan el más alto índice de su indicación y para lograr el éxito deben valorarse cuidadosamente todos los detalles. El proceso que debe seguirse es el siguiente esquematizado:

$1^{\circ}$ - Apreciar exactamente los requisitos de encajamiento, descenso, flexión, rotación, asinclitismo, posición y variedad de posición de la presentación.

$2^{0}$ - Apreciación de la dinámica uterina y comprobación de el cuello se ha hecho abdominal.

$3^{o}$ - Apreciación del estrecho inferior, características del pubis en previsión de cualquier anomalía pélvica clínicamente sospechable.

$4^{\circ}$ - Vitalidad fetal no comprometida y estado general de ia madre satisfactorio.

$5^{0}-$ Antes de decidirse a intervenir con el fórceps estimular la contracción uterina si fuere deficiente. 
$6^{\circ}$ - Intentar la rotación digital con la que puede lograrse la corrección de la deflexión lo que por sí solo puede resolver la distocia.

$7^{0}$ - En caso de no haber tenido éxito con las anteriores maniobras proceder a la aplicación del fórceps.

Sobra advertir las condiciones hospitalarias apropiadas y la debida preparación de la paciente (evacuación vesical, e intestinal, hidratación, sedación, anestesia).

\section{Qué técnica vamos a usar?}

$1^{\text {o }}$ - Técnica clásica. Maniobra de Scanzoni. Tiene los siguientes escollos:

a) La introducción de la rama posterior del fórceps puede perturbar la acomodación y convertir las O.P. en O.S.

b) $\mathrm{Al}$ efectuar la rotación bien sea de 135 grados (posteriores) o de 90 (transversas) esta rotación con las dos cucharas expone a desgarros vaginales.

c) Una vez rotada la presentación el fórceps queda invertido y se requiere nueva aplicación.

d) Mientras se hace esta nueva toma la cabeza ha podido desacomodarse nuevamente.

Tales inconvenientes de la maniobra clásica se han tratado de subsanar con múltiples maniobras entre las cuales vamos a mencionar algunas de las más conocidas:

Maniobra de De Lee. - (De la llave en la cerradura). Una vez aplicado el fórceps a la manera clásica se hacen sucesivas rotaciones previo desencajamiento y se desciende la presentación siempre en un grado menor al que se ha desencajado. En tal forma y reacomodando el fórceps, después de cada movimiento, se lleva la fontanela menor a la posición anterior o directamente a O.P. de donde se extrae con la técnica corriente.

Maniobra de Bill. --- (7) Es similar a la de De Lee porque desencaja la presentación pero la hace rotar en un solo tiempo.

Maniobra de fórceps invertido. - Se inicia la aplicación colocando la cuchara posterior correspondiente pero una vez en su sitio se desliza hacia el pubis al que debe pasar para hacerla an- 
terior, en donde queda invertida; la segunda cuchara se introduce en forma que case con la anterior para lo cual debe ocupar la extremidad posterior del diámetro que determina la cuchara anterior. Al hacer la rotación el fórceps queda al derecho. Esta es su ventaja pero se pueden hacer los otros reparos de la maniobra de Scanzoni.

Maniobra de Tatelbaun. - (8) Emplea dos fórceps, uno de rotación y otro de extracción. Para rotación emplea el fórceps no fenestrado, tipo Tucker y la extracción la hace con un fórceps fenestrado.

Técnicas similares se describen con el fórceps de Leff y con el fórceps de Kielland: el fórceps de Leff es un fórceps no fenestrado de cucharas muy cortas y angostas muy útil para la rotación y que por su naturaleza no sirve para la extracción.

Nuestra maniobra y similares posteriores a ella. - En 1950 describí la maniobra que más tarde presentaron en sendas tesis de grado los Dres. Isaías Arenas Buenahora y Luis Salazar Dávila. Fué acogida en España por Conil quien publicó su opinión en la Revista de Obstetricia y Ginecología en Buenos Aires. Algunos textos modernos como el de Dexeus la mencionan y describen sucintamente. Autores americanos la han usado y la utilizan con denominación de "shoe-horn" descrita por "Merchán". (9) Al propio tiempo se han publicado maniobras muy similares pero no idénticas, tales son la descrita por Frank Fara, Mash Steward, John V. Standard (10) quienes en 1959 publican una técnica en que utilizan el fórceps de Kyelland colocándolo exactamente como lo describimos nosotros pero sin rotar la cabeza; solamente con el objeto de colocar el fórceps en el diámetro anteroposterior de la madre.

Maughan (11) en 1956 hace una maniobra similar e inicia la rotación de la cabeza fetal pero deja la primera cuchara debajo del pubis la cual, dice, retiene la oreja correspondiente; luego desliza la cuchara posterior por el sacro y termina la rotación con ambas cucharas. Queda finalmente el fórceps al derecho.

King, (12) en 1951 modifica la técnica de Scanzoni en el sentido de no retirar sino una sola cuchara del fórceps cuando ha quedado invertido y la cuchara que queda, la desliza por la cara del feto hasta colocarla al derecho en la extremidad opuesta del diámetro que ocupaba inicialmente. Esto, con el objeto de 
que la cabeza no se devuelva a la posición inicial cuando se retiran las dos ramas al tiempo.

Otros autores reemplazan esta maniobra con la utilización de dos pares de fórceps del mismo tipo así que, cuchara invertida que retiran la reemplazan por otra que colocan al derecho.

La descripción simplificada de nuestra maniobra es la siguiente: para ODP y ODT úsese la mano derecha, cójase la rama izquierda invertida; colóquese posteriormente hacia el occipital; imprímase el movimiento de rotación hacia el pubis, rotándola simultáneamente con la cabeza. (Rotación en sentido de las agujas del reloj). La mano izquierda sirve de guía, ayuda a la rotación y el dedo índice va controlando el sitio que ocupa la fontanela menor. Una vez en el pubis la rama se desliza suavemente con pequeños movimientos ondulatorios hasta pasarla a ocupar la extremidad anterior del diámetro oblicuo izquierdo en donde se deja. La fontanela menor ha debido quedar en la extremidad anterior del diámetro oblicuo derecho. En seguida se coloca la rama posterior derecha a la manera clásica. Cuando la presentación está bien descendida puede suceder que la fontanela menor rote hacia el subpubis $\mathrm{OP}$, entonces, la rama anterior se lleva a ocupar la extremidad izquierda del diámetro transverso y la posterior, en el otro extremo. Los tiempos siguientes son los correspondientes a la extracción sencilla de las variedades anteriores.

Para las izquierdas posteriores e izquierdas transversas, se ejecutan los mismos movimientos pero a la inversa: se coge la rama derecha con la mano izquierda, la rama invertida, se guía con la mano derecha, se rota hacia el pubis (en este caso al inverso de las agujas del reloj) hasta pasarla a la extremidad anterior del diámetro oblicuo derecho. La rama posterior izquierda, según la técnica clásica. Obsérvese como en cada aplicación siempre una misma mano lleva las dos cucharas. La primera cuchara invertida y que, clásicamente no le correspondería a esa mano; y la segunda rama, dentro de las normas clásicas. (Fig. 4).

Casos especiales. - La técnica fué ideada para convertir las variedades posteriores y transversas en anteriores con la aplicación de la primera rama, teniendo en cuenta que antes de la rotación a $\mathrm{OP}$ es preciso completar el descenso por un diámetro oblicuo; pero como se mencionó atrás, si la presentación está bien 


\section{MANIOBRA DE RAMIREZ MERCHAN}

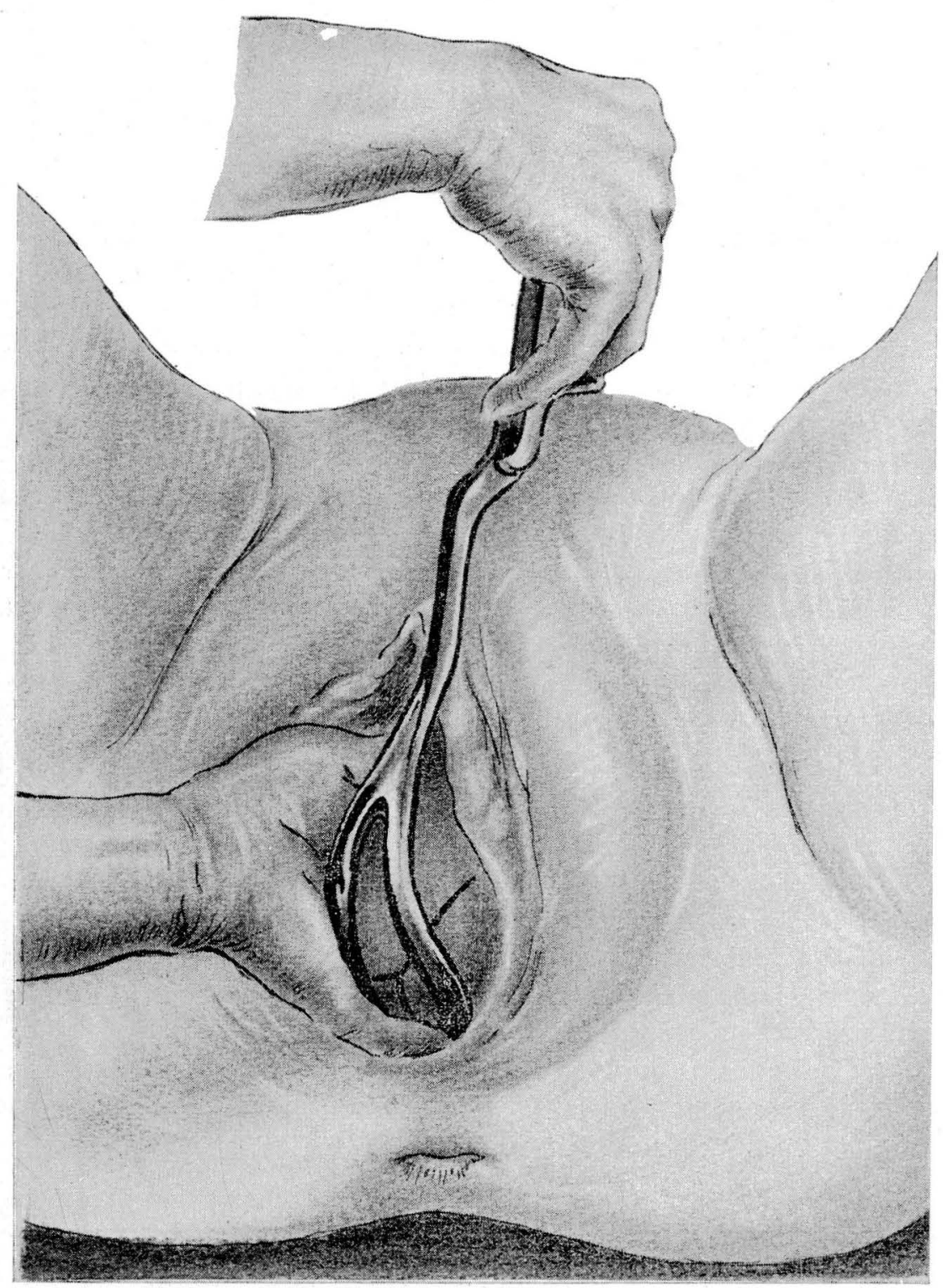

FIGURA № 1 

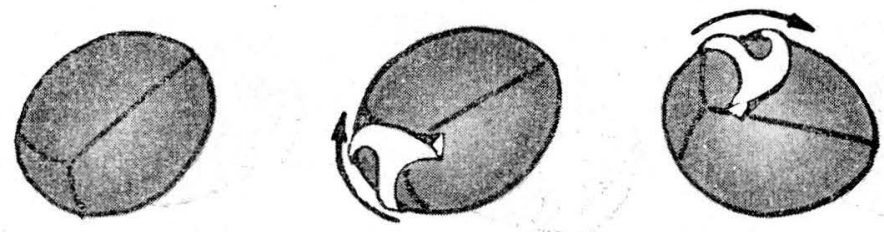

O D P

ODT

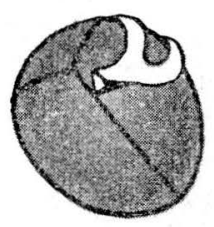

ODA

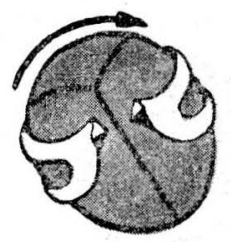

O D A

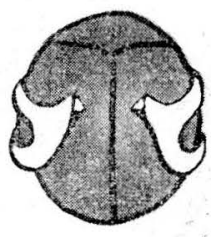

OP

APLICACION PARA OCCIPITO DERECHA POSTERIOR Fig 2 


\section{MANIOBRA DE RAMIREZ MERCHAN}
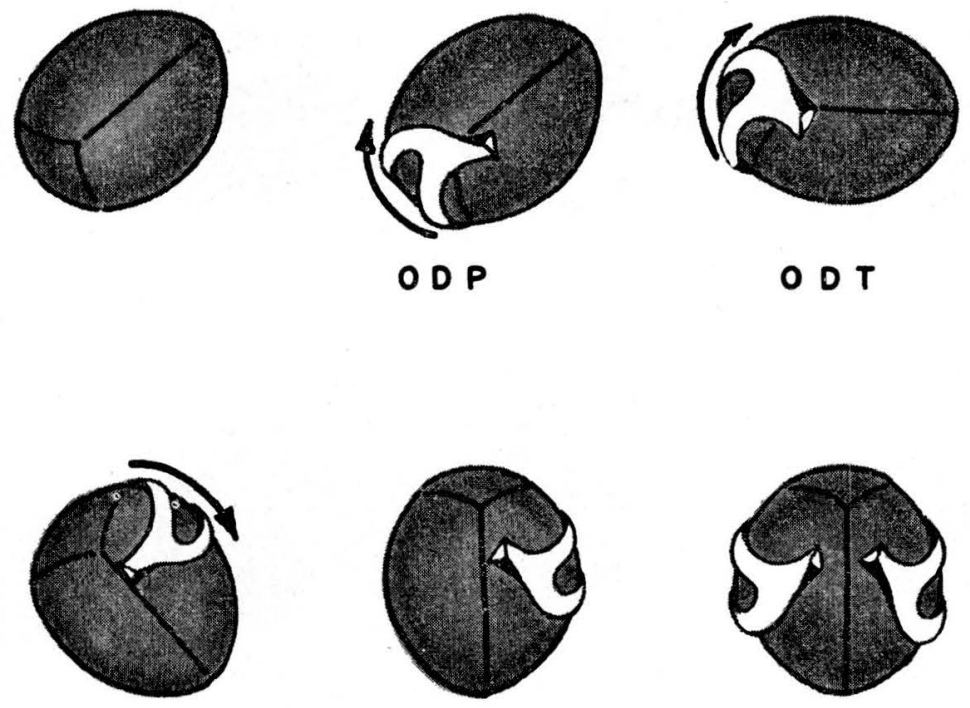

OD A

O P

APLICACION PARA OCCIPITO DERECHA POSTERIOR

Fig 3 
Vol. XII

$N^{\circ} 5$

MANIOBRA DE RAMIREZ MERCHAN
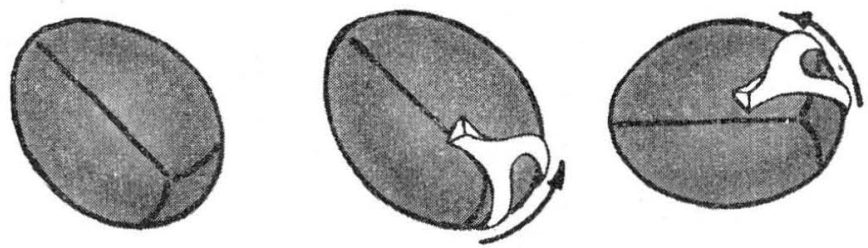

O I P

$01 \mathrm{~T}$
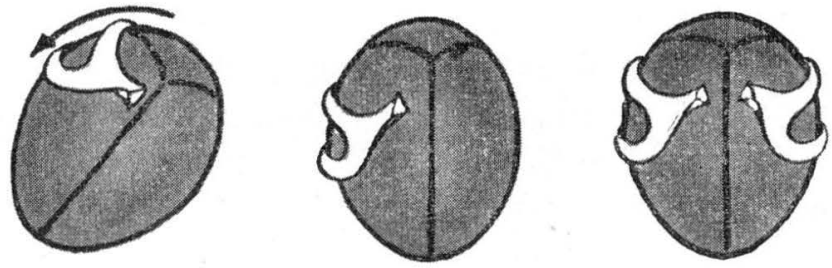

O IA

OP

APLICACION PARA OCCIPITO IZQUIERDA POSTERIOR

Fig 4 
descendida desde el prineipio puede hacerse la rotación a $\mathrm{OP}$, lo que simplifica ¿ún más el problema.

En algunas oportuni dades la presentación alcanza a pasar con la cuchara a la extremidad anterior del diámetro opuesto es decir, se trataba de hacer una DA y se ha llegado a una IA. En este caso la maniobra no ha fracasado, solo que la primera rama se deslizaría para hacerla posterior y la segunda rama, se colocaría con la técnica clásica en la extremidad anterior.

- Hemos observado también que cuando la cabeza no está encajada y se ensaya la técnica, lo más probable es que la presentación rote hasta ocupar la extremidad posterior opuesta; entonces la técnica no está indicada puesto que tampoco lo está el fórcops.

Las ventajas que hemos encontrado a esta maniobra se resumen así:

$1^{0}$ - Todas las variedades posteriores, así como las transversas en que está indicada, se transforman por efecto de la maniobra en variedades anteriores $\mathrm{y}$ a veces en variedades directas.

$2^{\circ}$ - Como consecuencia, siempre o casi siempre, se logran tomas ideales.

$3^{0}$ - La rotación efectuada con la cuchara, protegiendo el canal genital con la mano-guía, evita los desgarros vaginales, frecuentes cuando la rotación se hace con el fórceps articulado, según las maniobras instrumentales conocidas.

$4^{0}$ - El traumatismo para el feto es nulo desde el punto de vista de la maniobra misma.

$5^{0}$ - Solamente se hace una aplicación del fórceps y no se necesita sino el conocido modelo Simpson, en oposición a las maniobras hasta hoy propuestas que hacen doble toma, ya sea con un solo fórceps, como en la de Scanzoni, Bill-Scanzoni, KingScanzoni; o con dos fórceps, como en la maniobra de Tarnier Tucker, McLean, Leff, etc.

Para terminar considero conveniente insistir en que antes que elegir una maniobra. cualquiera que sea, y pretender resolver el caso con ella, es preciso valorarlo muy bien y sobre todo justipreciar la indicación del fórceps.

Tener en cuenta que la conformación pélvica puede hacer 
imposible el desprendimiento a pesar del encajamiento (pelvis infundibular); o puede exigir la rotación a occipito-sacra (pelvis plana) o ser más factible el desprendimiento por el diámetro oblicuo como ocurre en las pelvis androides y antropoides. (13)

En cuanto al instrumento mismo, aconsejamos en general el Simpson; pero para cada médico el mejor es el que sabe manejar con más propiedad, de acuerdo con los requisitos y condiciones de la época.

\section{BIBLIOGRAFIA}

(1) KENNETH BOWES Recientes Avances en Obstetricia y Ginecología. Ediciones Toro S. A. Pág. 295.

(2) PLATA RUEDA ERNESTO Traumatismo Obstétrico en el recién nacido. Tesis de grado. Fac. de Medicina U. Nal. 1950.

(3) DE LEE GREENHILL Principios y Práctica de Obstetricia. Versión al Castellano de la $7^{a}$ Edición por Oscar Carrera. Edit. Hispanoamericana. Méjico.

(4) LULL and KINGBROUGH Clinical Obstetrics. J. B. Lippincott Company. 1953.

(5) DE LEE GREENHILL Op. Cit.

(6) DUBRISAY JEANNIN Praecis D'acouchement. huitiemme. Edition 1936.

(7) LULL and KINGBROUGH Op. cit.

(8) TAtelbaum ABRAHAM J. A Metod of Forceps rotation $m$. post. pos. Pág. 553. Am. J. Of. Obs. and Cyn. III. 1959.

(9) RICHARD A. KUTCIPAL The persist. occiput Posterior Position. Obstetrics and Gyn. Vol. 14 Ni 3. Pág. 296.

(10) Year Book of Obst. and Gyn. 1956. 1957. Pág. 174.

(11) MAUHAN G. B. The Safe and simple Delivery of Persistent Posterior and Transverse Position. Am. J. of Obst. and Gyn. Vol. 71 N: 4. Pág. 741.

(12) E. L. KING, J. A. KING, etc. The Modification of the Scanzoni rotation in the management of persistent occipitoposterior positions. Am. J. of. Obst. and Gyn. Vol. 61 N: 4 Pág. 872

(13) EDWARD H. DENNEN Choice of Forceps. Clinical Obst. and Gyn. Junio 1959. Pág. 367. 